\title{
Mechano-transduction via the pectin-FERONIA complex regulates ROP6 GTPase signaling in Arabidopsis
}

${ }^{1}$ FAFU-UCR Joint Center for Horticultural Biology and Metabolomics Center, Haixia Institute of Science and Technology, Fujian Agriculture and Forestry University, Fuzhou, Fujian, China

${ }^{2}$ Institute of Integrative Genome Biology and Department of Botany and Plant Sciences, University of California, Riverside, CA 92521

*Correspondence to: Zhenbiao Yang (yang@ucr.edu).

\section{SUMMARY}

During growth and morphogenesis, plant cells respond to mechanical stresses resulting from spatiotemporal changes in the cell wall that bear high internal turgor pressure. Microtubule (MT) arrays are re-organized to align in the direction of maximal tensile stress to guide the synthesis of cellulose, reinforcing the local cell wall. However, how mechanical forces regulate MT re-organization remains largely unknown. Here, we demonstrate that mechanical signaling that is based on the CrRLK1L subfamily receptor kinase FERONIA (FER) regulates the reorganization of cortical MT in cotyledon epidermal pavement cells (PC) in Arabidopsis. Recessive mutations in FER compromised MT response to mechanical perturbations such as single cell ablation, compression and Isoxaben treatment in these pavement cells. These perturbations promoted the activation of ROP6 GTPase that acts directly downstream of FER. Furthermore, defects in the ROP6 signaling pathway negated the reorganization of cortical MTs induced by these stresses. Finally, reduction in highly demethylesterified pectin, which binds the extracellular malectin domain of FER and is required for FER-mediated ROP6 activation, also impacted mechanical induction of cortical MT reorganization. Taken together our results suggest that the FER-pectin complex senses and/or transduce mechanical forces to regulate MT organization through activating the ROP6 signaling pathway in Arabidopsis. 


\section{INTRODUCTION}

Cellular organisms experience external forces as well as internal mechanical cues, which need to be integrated with chemical signals to coordinate cell behaviors in space and over time. Plant cells are under tremendous mechanical stresses from strong internal turgor pressure. As plant cells are attached to each other via the cell wall, supracellular patterns of physical forces are generated during growth, causing large tension in the epidermis. The resulting forces may provide a coordinating supracellular signal to regulate cell division and cell growth/expansion and plant morphogenesis from the cell to organ level [1-4]. Changes in growth pattern and cell expansion requires corresponding local or global modification of the cell wall, resulting in spatiotemporal changes in mechanical stresses from turgor pressures. Plant cells must monitor these mechanical changes to assure the cell wall homeostasis and integrity and to coordinate with cell growth and morphogenesis $[5,6]$. The MSL family members have been shown to monitor osmotic shocks for the maintaining of cell viability [7, 8], while CrRLK1L members might be candidates for the sensors that monitor mechanical forces for cell wall modification in plants $[5,9]$. However, direct evidence for their mechanosensing capacity is lacking, and thus the molecular mechanisms for mechanical sensing and signaling in plants remain poorly understood.

As in animal cells, the cytoskeleton plays an important role in mechanical responses in plant cells. Cortical microtubules (MT) were found to align with maximal stress in plants $[10,11]$. Increasing evidence supports a role for mechanical forces in the regulation of MT orientation in plant cells [12-17]. MT in turn direct the synthesis of cellulose to reinforce the cell wall in countering the mechanical stress. Pavement cells (PC) in the cotyledon and leaf epidermis provide an attractive system for studying MT-based mechanical feedback regulation of cell morphogenesis [18]. In most angiosperms, leaf epidermal PC exhibit jigsaw puzzle shapes with interdigitated indention and lobe-like outgrowths in the anticlinal direction. Two distinct, but not necessarily exclusive, models have been proposed to initiate the interdigitated cell shape pattern. Auxin is thought to be the chemical signal, as it is important for and promotes the lobing of PCs by activating ROP/Rac GTPase-dependent local cytoskeletal reorganization [19-24]. In particular, cortical MTs enriched in PC indentation regions guide localized deposition of stiff cellulose microfibrils (CMF), thus inhibiting outgrowth $[20,22,25-28]$, and this localized MT organization is promoted by katanin-based MT severing promoted by ROP6, which is activated by cell surface auxin sensing $[22,24,29]$.

In parallel or consequently, mechanical stress may trigger a MT-based feedback mechanism for the cellulose-based cell wall reinforcement, and thus the maintenance of pavement cell shape $[18,30]$. This stress-induced positive feedback loop may be initiated by auxin/ROP pathway described above. In an alternative non-exclusive scenario, mechanical and chemical polarities may emerge early on in the anticlinal walls of PC, before they start waving [31]. Mechanical forces were recently found to modify MT organization in leaf PC [32]. It has been proposed that stress in the outer wall may amplify such initial deformations $[31,33]$, thereby maintaining mechanical stress at a relatively low level, while increasing 
cell volume [34]. Identification of the unknown molecule that senses the mechanical stress would help to understand how the mechanical signal activates MT reorganization and whether it acts independently from or coordinately with the auxin signal.

FER (Feronia), CrRLK1Ls family (Catharanthus roseus RLK1-like kinase family) [35], regulates multiple processes during plant growth, such as plant immunity [36-38], cell elongation [39, 40], root and root hair growth [41, 42], cell morphogenesis [43] and seed development [44]. Most of these processes are linked to cell wall homeostasis and/or cell wall integrity (CWI) maintenance. Moreover, FER is required for mechanically induced changes in $\mathrm{Ca}^{2+}$ [4]. CrRLK1L members contain two extracellular carbohydrate-binding malectin (Mal) domains [35]. Feng et al recently showed that FER binds pectin in vitro through its extracellular domain and maintains cell-wall integrity during salt stress [45]. Furthermore, Lin et al showed that FER senses highly demethyesterified pectin to directly activate the ROP6 signaling pathway during PC morphogenesis in Arabidopsis [46] (accompanying manuscript). Here we show that FER participates in the sensing and/or signaling of mechanical stress that activate the ROP6 signaling pathway to induce MT rearrangement in Arabidopsis cotyledon epidermis.

\section{RESULTS}

\section{The fer-4 Mutation Compromises Sensitivity to Mechanical Stress-induced MT Rearrangement}

Recessive fer alleles exhibit severe pavement cell (PC) shape defects, such as wider indentation, less shallow lobes, indicative of weaker cell wall in the indentation regions of these cells $[43,46]$. Given demethylesterified pectin's binding to and requirement for the activation of FER [46], the cell wall reinforcement may be mediated by FER's sensing of mechanical stress via its interaction with pectin. In animals, extracellular matrix (ECM)integrin (ECM receptor) plays an important role in sensing mechanical forces [47]. Binding of the cell wall to Wsc1 is also proposed to be essential for its sensing of mechanical stress in yeast [48-51]. Here we tested whether FER is involved in sensing the mechanical stress using a MT re-organization assay, as the reorganization of cortical MT (CMT) has been used as a readout for mechanical stress [17, 30]. Cortical MT organization into ordered arrays is indicated by MT anisotropy measured by FibrilTool, an ImageJ plug-in to quantify fibrillar structures in raw microscopy images [52]. MT has often been labeled with fluorescence protein tagged-tubulin, but we found GFP-tubulin's labeling of cortical MTs in fer-4 PCs was quite weak, making the measurement of MT anisotropy quite difficult. Thus we used GFP-MAP4, which gives clear labeling of MTs both in wild type and fer mutants. As shown previously with GFP-tubulin labeled MT, mechanical stress induced a dramatic increase in the anisotropy of MT labeled with GFP-MAP4. MT anisotropy in fer-4 PCs (cultured in $1 / 2$ MS with $1 \%$ agar) was reduced to nearly $50 \%$ (from $0.058 \pm 0.013$ to $0.034 \pm 0.010)$ in the indentation region when compared to wild type PC, consistent with wider indentation regions in fer-4 PC (Figure S1) [46]. 


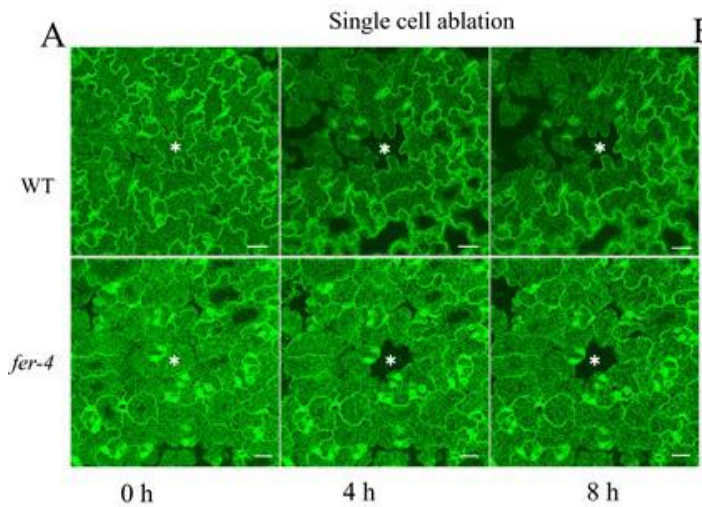

B

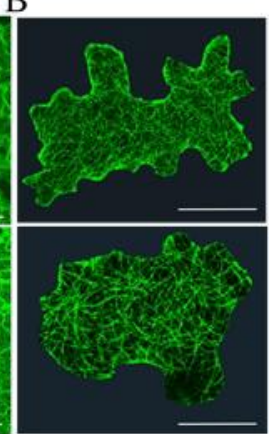

$0 \mathrm{~h}$
Single cell ablation

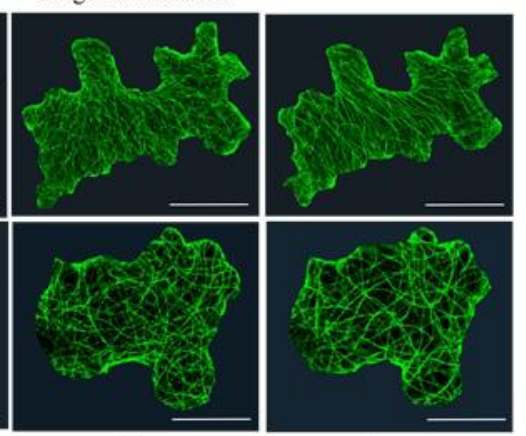

$4 \mathrm{~h}$

$8 \mathrm{~h}$

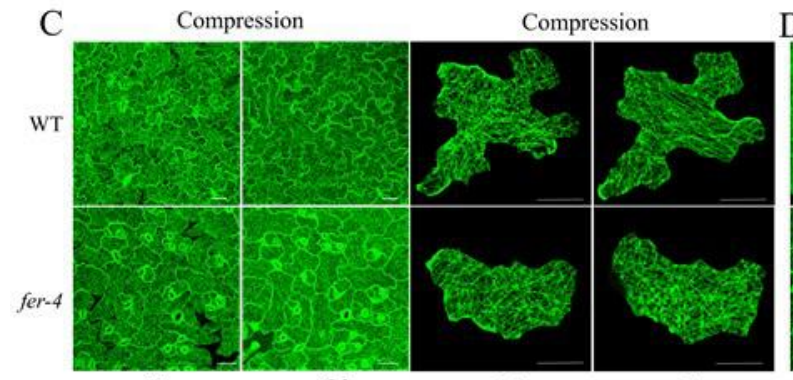

$0 \mathrm{~h}$

$6 \mathrm{~h}$

$0 \mathrm{~h}$

$\mathrm{F}$

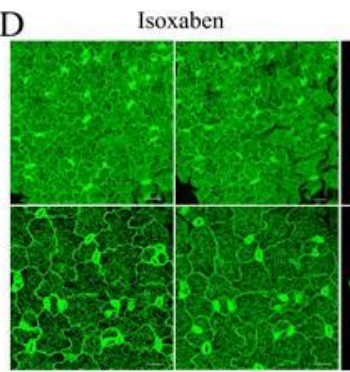

$0 \mathrm{~h}$

$6 \mathrm{~h}$

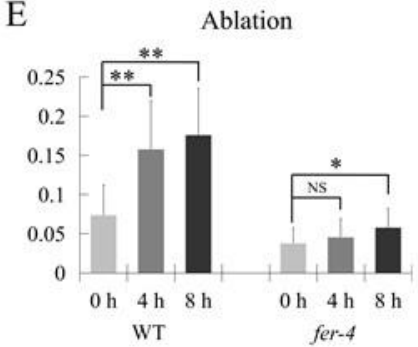

Compression

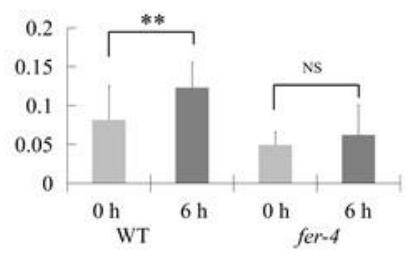

G

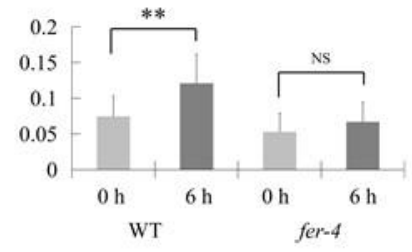

Figure 1. fer-4 PCs exhibit reduced sensitivity to mechanical stresses in inducing MT rearrangement. Cotyledons from Col-0 (WT) and fer-4 expressing GFP-MAP4 were subject to different mechanical stresses induced by single cell ablation (A and $B$ ), compression (C) and isoxaben treatment (D) and cortical MT anisotropy were measured (E, $\mathrm{F}$, and $\mathrm{G}$, respectively).

(A) MT rearrangement after single cell ablation treatments on 3 DAG (days after germination) cotyledon PCs from WT (GFP-MAP4) and fer-4 (fer-4 x GFP-MAP4). In WT, ablation of single PC resulted in the circumferential distribution of MT arrays around the site of physical perturbations (White asterisk marks site of ablation). In fer-4 mutant, MT rearrangement induced by single cell ablation nearly lost. Scale bar $50 \mu \mathrm{m}$.

(B) Representative PCs from A were enlarged to show clear MT arrangements. Scale bar, 50 $\mu \mathrm{m}$.

126 (C) MT rearrangement after 6 hrs compression treatment on WT and fer-4 3 DAG cotyledon PCs. Representative individual cells are shown in the right panels. Scale bar, $50 \mu \mathrm{m}$. cotyledon PCs. Representative individual cells are shown in the right panels. Scale bar, 50 $\mu \mathrm{m}$. 
131 (E) Quantification of MT Anisotropy in A. Two layers of PCs surrounding the ablated cell were used for quantification. Data are mean degrees from $>20$ independent cells \pm SE. Asterisks indicate the significant difference $(* p<0.05, * * p<0.01$, NS, no significant difference, Student's $t$-test) between the wild type and the mutants in the above assays. (just below the tip) were quantified. Data are mean degrees from $>20$ independent cells \pm SE. Asterisks indicate the significant difference $(* p<0.05, * * p<0.01$, NS, no significant difference, Student's $t$-test) between the wild type and the mutants in the above assays.

To assess whether FER regulates microtubule response to mechanical stress, external mechanical forces generated by ablation, compression and Isoxaben treatments were applied on cotyledons to alter stress patterns [30]. Firstly, we carried out single cell ablation by high-dosage UV light radiation on the epidermis of cotyledons from seedlings cultured in $1 / 2$ MS with $1.0 \%$ agar. Maximal stress directions are predicted to become circumferential to the ablation site independent of cell geometry [30]. In WT, 4-8 hours after ablation, the orientation of cortical MT arrays in PCs surrounding the ablation site changed to a circumferential rearrangement (Figure 1A), as reported previously [30]. The anisotropy of cortical MT was greatly increased (from $0.07 \pm 0.038$ to $0.16 \pm 0.061$ and $0.18 \pm 0.060$ at 4 and $8 \mathrm{hrs}$ after ablation, respectively). However, when a single PC was ablated in fer-4 cotyledons, the anisotropy was only slightly increased from $0.038 \pm 0.019$ to $0.046 \pm 0.023$ and $0.058 \pm 0.024$ at 4 and $8 \mathrm{hrs}$, respectively (Figures $1 \mathrm{~A}, 1 \mathrm{~B}$ and 1E). Thus, ablation induced MT rearrangement was essentially lost in the fer-4 mutant.

Application of compressive forces resulted in an increase in overall stress and hyperalignment of MT [30]. We directly applied compressive forces to PCs by using a coverslip pressed on the surface of the cotyledons and kept in place using adhesive silicone applied on the margins [32]. The rearrangement of cortical MT became more aligned by $6 \mathrm{hrs}$ in PCs from WT cotyledons but not from fer-4 (Figures $1 \mathrm{C}$ and $1 \mathrm{~F}$ ). Isoxaben, which inhibits cellulose synthesis and thus increases tensile stress on the cell wall, was shown to induce hyper-bundling and hyper-alignment of cortical MT along the predicted directions of maximal stress in shoot meristem cells [53]. Treatment of 3-day-old seedlings with $40 \mu \mathrm{M}$ isoxaben for $6 \mathrm{hrs}$ led to a sizeable increase in the anisotropy of MT (Figures 1D and 1F). In fer-4 mutant, MT kept randomly aligned 6 hrs after the treatment (Figures 1D and 1F).

\section{Mechanical stresses promote ROP6 GTPase activation}

Katanin-mediated MT severing is required for MT responses to mechanical stresses in the shoot apical meristem and leaf PC in Arabidopsis [30, 54]. In Arabidopsis PCs, cortical MT organization is regulated by the ROP6 signaling pathway acting upstream of katanin [22, 29], and FER activates ROP6 directly via their interactions with RopGEF14 [46]. Thus, we hypothesized that mechanical stress activates the ROP6 pathway via FER to promote the katanin-mediated MT rearrangement. To test this hypothesis, we first determined whether ROP6 activation is mediated by mechanical stress. We treated 7 DAG (days after germination) GFP-ROP6 seedlings [24] with $40 \mu \mathrm{M}$ Isoxaben, which induces an external mechanical stress overriding the internal developmental stress pattern. The amount of active 
GFP-ROP6 protein increased by $50-100 \%$ in $1 \mathrm{~h}$ to $2 \mathrm{~h}$ after Isoxaben treatments compared with untreated control $(0 \mathrm{~h}$, Figure $2 \mathrm{~A})$. This effect is specific for ROP6, because the amount of active GFP-ROP2 protein was not significantly affected by the same treatment in GFP-ROP2 transgenic seedlings (Figure 2B). Different concentrations of Isoxaben will generate different strengths of mechanical forces. We found that 10-40 $\mu \mathrm{M}$ Isoxaben were the optimal concentrations for the induction of ROP6 activity (Figure S3A). We confirmed that these concentrations of Isoxaben induced cell shape changes (Figure S2).

To directly test whether ROP6 activity is induced by mechanical stress, we next assessed the effect of externally applied compression on ROP6 activity. Compression was applied on 3-weeks old leaves. Four glass slides were placed on one half of a leaf as compression treatment, and the other half was free of external pressure as control. At various times, the glass slides were removed, and the leaf was dissected into two halves and immediately frozen in liquid nitrogen for ROP6 activity assay. The compression induced an increasing ROP6 activation over the times of treatment (Figure 2C). After 2 hours of compression, the amount of active GFP-ROP6 increased to about 2 times compared with the untreated control $(0 \mathrm{~h}$, Figure $2 \mathrm{C})$. When compression was applied on Col-0 leaves, the activation of native ROP6 also detected after 2 hours of compression (Figures S3B and S3C). These results clearly demonstrate that mechanical stresses promote ROP6 activation.

A

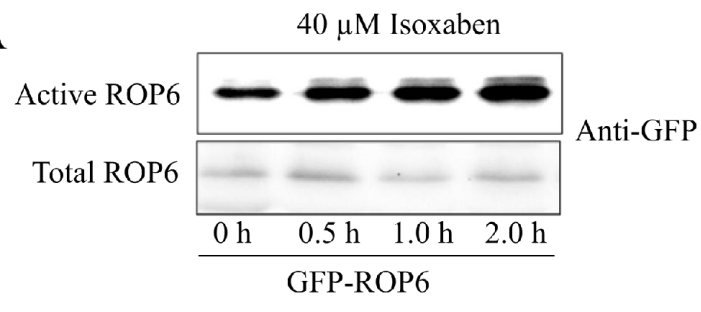

B

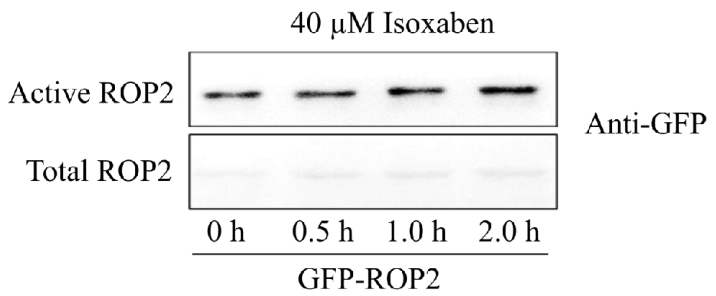

C

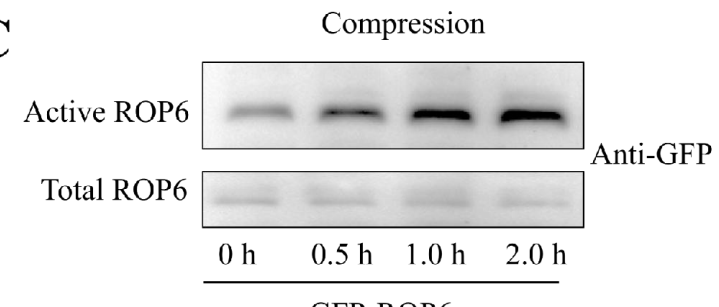

193

194

195

196

197
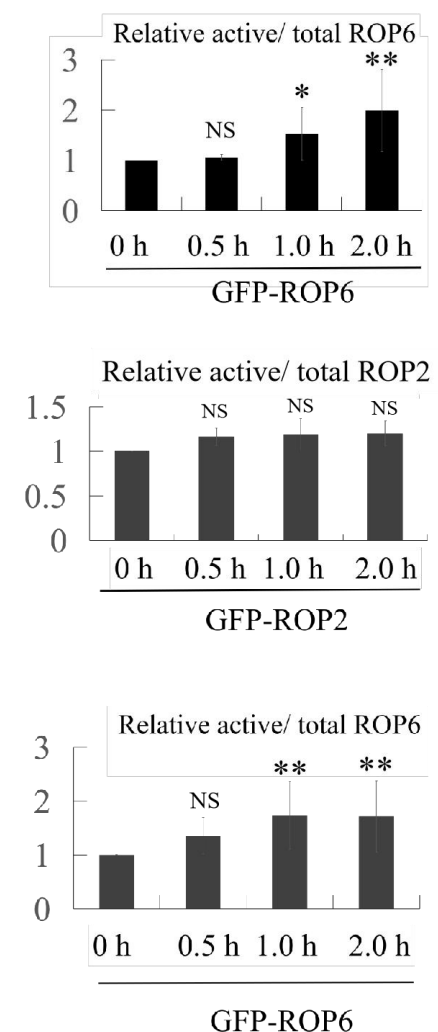

Figure 2. Mechanical stresses induce the activation of ROP6 but not ROP2.

(A) Western blots based ROP6 activity measurement in 7 DAG seedlings of GFP-ROP6 with isoxaben treatments in different times. ROP6 activation by Isoxaben was analyzed by pull-down assay, as described previously [27]. Quantification of relative active GFP-ROP6 
198 level (amount of GTP-bound GFP-ROP6 divided by amount of total GFP-ROP6) to control 199 (as "1") is shown. Data are mean activity levels from three independent experiments \pm SD. $200 * \mathrm{p}<0.05, * * \mathrm{p}<0.01$, NS, no significant difference, Student's $t$-test.

201 (B) ROP2 activity in GFP-ROP2 with and without isoxaben treatments in different times. 202 ROP2 activity can be slightly induced by $40 \mu \mathrm{M}$ Isoxaben. Data are mean activity levels 203 from three independent experiments \pm SD. NS, no significant difference, Student's $t$-test.

204 (C) ROP6 is activated by compression. ROP6 activity in 3 weeks leaves of GFP-ROP6 with compression treatment in different times. Data are mean activity levels from three independent experiments \pm SD. ${ }^{*} \mathrm{p}<0.01, \mathrm{NS}$, no significant difference, Student's $t$-test.

\section{FER and RopGEF14 Are Required for the ROP6 Activation Induced by Mechanical Stress}

210 We next determined whether FER is required for the activation of ROP6 induced by mechanical stress. GFP-ROP6 and fer-4x GFP-ROP6 lines were used for Isoxaben and compression treatments as described above. In contrast to a 1-fold increase in the amount of active GFP-ROP6 after 7 DAG GFP-ROP6 seedlings were treated with Isoxaben for 1-2 hrs, no significant increase in GFP-ROP6 activity was observed in fer-4xROP6-GFP seedlings with the same treatments (Figure 3A). Similar results were obtained when ROP6 activity was assayed in Col-0 and fer-4 lines using anti-ROP6 to detect ROP6 (Figure S4A).

We also investigated whether FER is required for the ROP6 activation induced by compression in 3 weeks old GFP-ROP6 and fer-4xGFP-ROP6 leaves. As shown in Figure 3, $2 \mathrm{hrs}$ of compression induced GFP-ROP6 activation by two folds compared with mock treatment in GFP-ROP6 leaves. However, in fer-4xGFP-ROP6 leaves, the amount of active ROP6 decreased in the control, and compression failed to induce ROP6 activation (Figure 3B).

FER activates ROP6 signaling through direct interaction with the ROP6 activator ROPGEF14 in the cotyledon epidermis [46]. Therefore, ROPGEF14 is expected to be required for mechanical stress induced ROP6 activation. To confirm this, Isoxaben and compression were applied on the 7-day old seedlings and 3 weeks-old leaves from the gef14-2xGFP-ROP6 and GFP-ROP6 lines [46], respectively. As expected, the ropgef14-2 knockout mutation greatly compromised GFP-ROP6 activation induced by both Isoxaben and compression (Figures 3C and S4B). Taken together, we show that the FER-ROPGEF14 complex is required for the induction of ROP 6 activation by mechanical forces. 


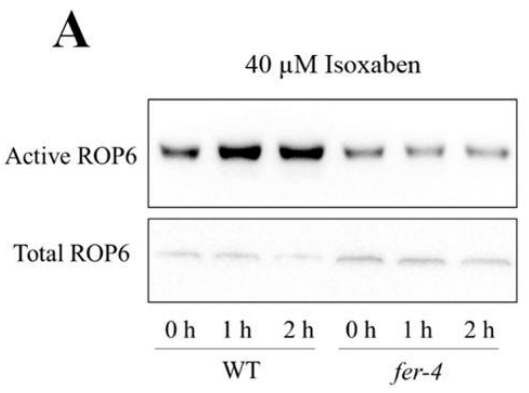

Relative active/total ROP6

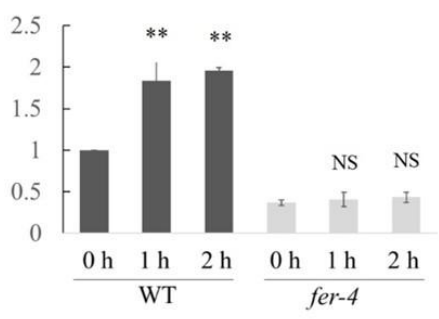

B

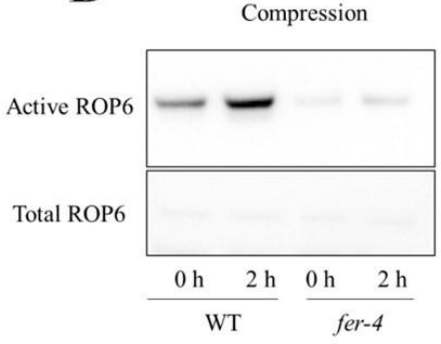

Relative active/total ROP6

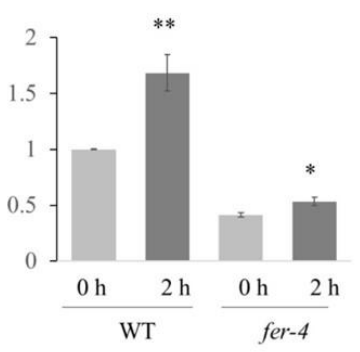

C

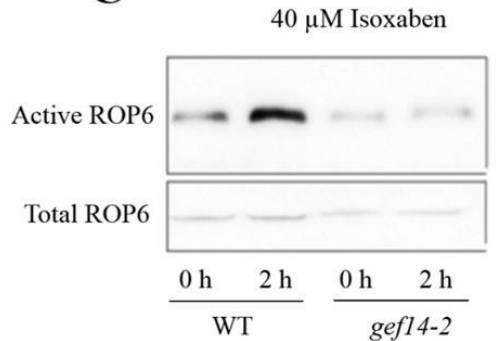

Relative active/total ROP6

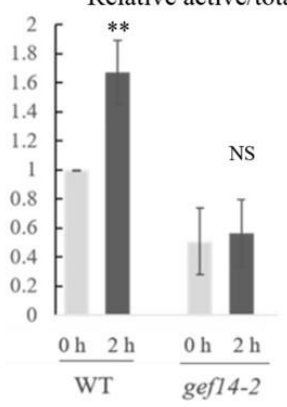

Figure 3. FER is required for mechanical stress on ROP6 activation.

(A) ROP6 activity in 7 DAG seedlings of GFP-ROP6 and fer-4 $x$ GFP-ROP6 with and without isoxaben treatments for different times. ROP6 activity can be induced by $40 \mu \mathrm{M}$ Isoxaben in GFP-ROP6 but not in fer-4 $x$ GFP-ROP6. Data are mean activity levels from three independent experiments $\pm \mathrm{SD}$. **, $\mathrm{P}<0.01$; NS, no significance

(B) Compression induced ROP6 activation is lost in fer-4. Compression was applied on leaves from 3 weeks old control (GFP-ROP6) and fer-4 (fer-4 $x$ GFP-ROP6) plants. After 2 hrs of compression, leaves were loaded for ROP activity measurement. Data are mean activity levels from three independent experiments $\pm \mathrm{SD}$. *, $\mathrm{p}<0.05 ; * *, \mathrm{P}<0.01$

(C) ROPGEF14 is required for Isoxaben induced ROP6 activation. ROP6 activity in 7 DAG seedlings of WT and gef14-2 with $40 \mu \mathrm{M}$ isoxaben treatments for 2 hours. ROP6 activity can be induced by $40 \mu \mathrm{M}$ Isoxaben in WT but not in gef14-2. Data are mean activity levels from three independent experiments $\pm \mathrm{SD}$. **, $\mathrm{P}<0.01$; NS, no significance

\section{ROP6 Signaling Is Required for the MT Rearrangement Induced by Mechanical Stress}

Because FER-dependent mechano-transduction activates MT rearrangement and ROP6 signaling, which was previously shown to promote the organization of cortical MT in PCs [22], it is reasonable to hypothesize that the FER-based mechano-transduction regulates MT rearrangement via ROP6 signaling. To test this hypothesis, we investigated whether ROP6 signaling is required for mechanical stress induced MT rearrangement. We previously showed that RopGEF14 and RIC1 act as ROP6 activator and effector to regulate MT organization, respectively [22, 46]. Thus, three mutants gef14-2, rop6-1 and ricl-1 were 
256 used to assess the role of ROP6 signaling in the mechanical induction of MT rearrangement. 257 Unlike fer-4, all these three mutants only exhibit relatively mild defects in PC 258 morphogenesis, likely due to the existence of another ROP or signaling pathway that is 259 functionally overlapping with ROP6 in the promotion of MT ordering during PC 260 morphogenesis. Thus, we expected these mutants to be less compromised in the response to 261 external forces than fer-4. Indeed 3 DAG cotyledons from gef14-2, rop6-1 and ricl-1 mutants all appeared to have normal sensitivity to ablation-induced MT rearrangement when seedlings were cultured in $1 / 2$ MS medium with $1 \%$ agar (Figure S5). We reasoned that lowering tensile stress would reduce the response of MT rearrangement to mechanical stress and thus condition the gef14-2, rop6-1 and ric1-1 mutants to be less sensitive to the weaker mechanical stress. Older cotyledons, which have thicker and stronger cell wall, are expected to experience less internal tensile stress. In addition, cell wall tension is reduced when seedlings are cultured in higher concentrations of agar $(2.5 \%)$ than in regular agar concentration (1\%) [17]. Therefore, we cultured gef14-2, rop6-1 and ricl-1 mutants to 7 DAG in both regular and high concentrations of agar (1\% and $2.5 \%)$. We found that the gef14-2 mutation greatly reduced the sensitivity to single cell ablation in inducing MT rearrangement in $7 \mathrm{DAG}$ cotyledons under normal (1\%) agar conditions (Figures 4A and 4C). Both rop6-1 and ricl-1 mutants exhibited less response to ablation when cultured in $1 / 2$ MS with $2.5 \%$ agar (Figures $4 \mathrm{~B}$ and 4D). To further confirm the importance of the ROP6 signaling pathway in mechanical stress-induced MT rearrangement, we incubated 7 DAG gefl4-2, rop6-1 and ricl-1 in high concentration of agar (2.5\%) in $1 / 2 \mathrm{MS}$ with $40 \mu \mathrm{M}$ Isoxaben. MT rearrangement were induced and the anisotropy value ratio $(4 \mathrm{~h} / 0 \mathrm{~h})$ was more than 2 in WT, whereas in gef14-2, rop6-1 and ric1-1 the anisotropy value ratios (4h/0h) were less than 1.5 (Figures $4 \mathrm{E}$ and $4 \mathrm{~F}$ ). Taken together, the ROP6 pathway is required for MT organization response to mechanical stress. 


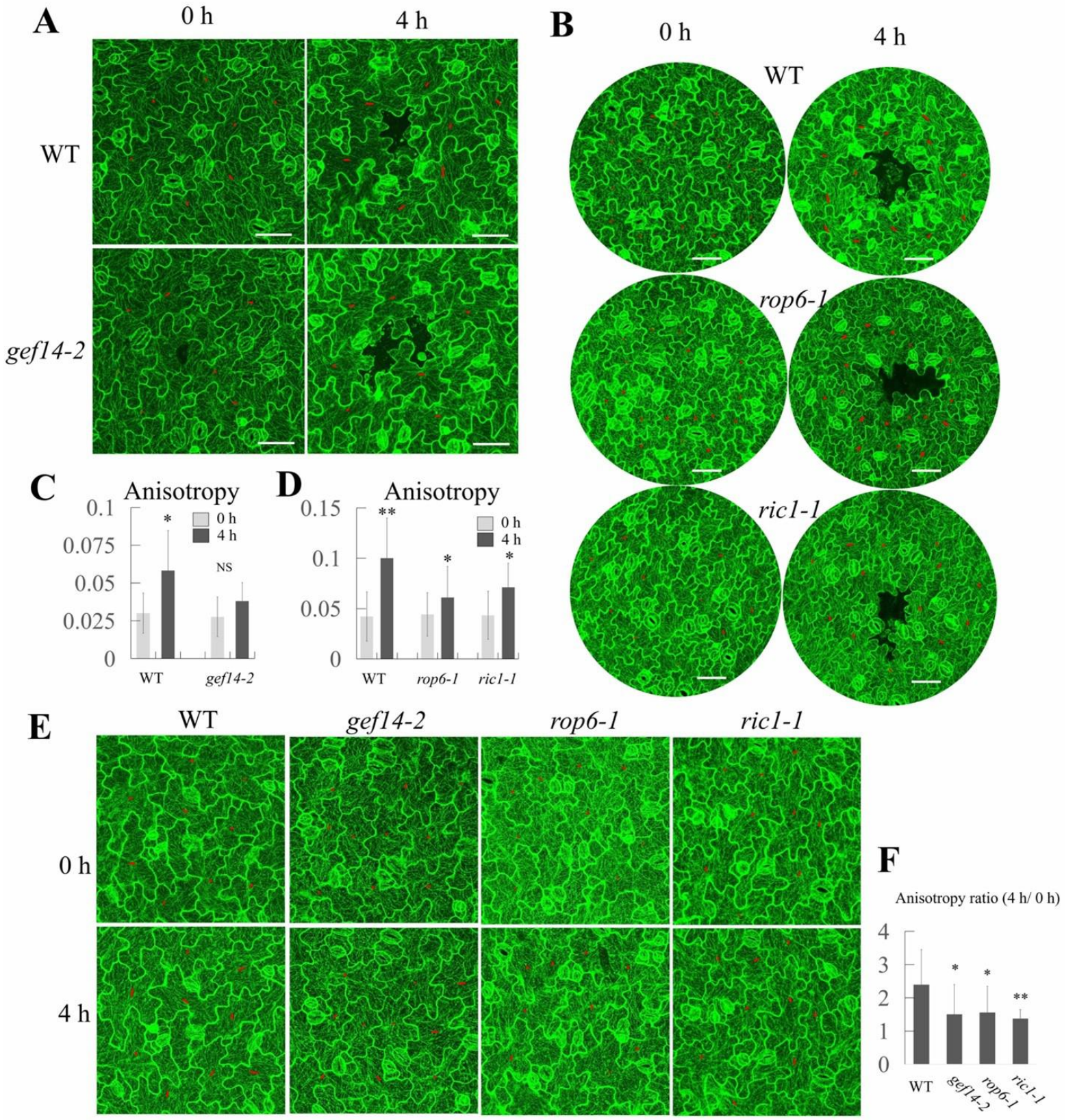
(A) MT rearrangements after single cell ablation in WT and gef14-2 PCs. 7 DAG cotyledons grown in $1 / 2$ MS with normal concentration of agar $(1.0 \%)$ were observed by confocal microscopy. Red line represents the anisotropy of MT. Two layers of PCs around ablation point were quantified. Bar $=50 \mu \mathrm{m}$.

289 (B) MT rearrangements after ablation in WT, rop6-1 and ric1-1 PCs. 7 DAG cotyledons grown in $1 / 2$ MS with high concentration of agar $(2.5 \%)$ were observed in confocal 291 microscopy. Red line represents the anisotropy of MT. Two layers of PCs around ablation 292 point were quantified. $\operatorname{Bar}=50 \mu \mathrm{m}$. 
293 (C) Anisotropy values of MT in (A). gef14-2 was less sensitive to ablation-induced anisotropy increase. Data are mean degrees from $>50$ independent cells \pm SE. *, $p<0.05$; NS, no significance (D) Anisotropy value of MT in (B). Both rop6-1 and ricl-1 were less sensitive to ablationinduced anisotropy changes compared to WT. Data are mean degrees from $>50$ independent cells \pm SE. $*, p<0.05 ; * *, p<0.01$.

(E) MT rearrangements after $40 \mu \mathrm{M}$ Isoxaben treatment in WT, gef14-2, rop6-1 and ricl-1 PCs. 7 DAG cotyledons grown in $1 / 2$ MS with high concentration of agar $(2.5 \%)$ were observed by confocal microscopy. Red line represents the anisotropy of MT. PCs at the same position of cotyledon were quantified. Bar, $50 \mu \mathrm{m}$.

(F) Anisotropy value ratios (4 h / $0 \mathrm{~h})$ of MT in E. gef14-2, rop6-1 and ric1-1 were less sensitive to $40 \mu \mathrm{M}$ Isoxaben treatment in the induction of anisotropy change compared to WT. Data are mean degrees from $>50$ independent cells \pm SE. B. ${ }^{*}, \mathrm{p}<0.05 ;{ }^{* *}, \mathrm{p}<0.01$

\section{Highly Demethylesterified Pectin Is Required for ROP6 Signaling Induced by Mechanical Stress}

The connection between a transmembrane receptor and extracellular matrix provides a common mechanism for sensing mechanical forces in animal and fungal cells [55]. FER binds highly demethylesterified pectin through its extracellular MALA domains to ROP6 signaling in during PC morphogenesis [46]. Indentation regions of the anticlinal wall in PC are enriched in highly demethylesterified pectin [46] and undergo highest mechanical stress [30]. Thus, it is reasonable to propose that the FER-pectin connection senses the mechanical stress in these indentation regions, generating a positive feedback regulation of ROP6 signaling and MT rearrangement and subsequent microfibril deposition for the reenforcement of the indentation regions. To test this hypothesis, we investigated whether highly demethylesterified pectin is required for mechanical force sensing. To assess the impact of pectin demethylesterification levels, we used a transgenic line overexpressing pectin methylesterase inhibitor 1 (PMEI1 OE), which contains reduced pectin demethylesterification [46]. First, we performed single cell ablation on 3 DAG cotyledon epidermis of the PMEI1 OE line. Four hrs after ablation, cortical MT of PCs became greatly rearranged in WT (Figure 5A). However, compared to WT, PMEI1OE PCs showed less response to ablation (Figure 5A). In WT, the anisotropy was increased nearly by 2 folds from $0.041 \pm 0.007(0 \mathrm{hr})$ to $0.116 \pm 0.026$ at $4 \mathrm{hrs}$ after ablation. In contrast, in PMEI1OE the anisotropy was increased by one fold from $0.031 \pm 0.006(0 \mathrm{~h})$ to $0.066 \pm 0.011$ (4 h; Figure $5 \mathrm{~B})$.

Next we investigated whether pectin demethylesterification affects ROP6 activation induced by mechanical stress. ROP6 activity in seedlings of WT and PMEI1OE with and without $40 \mu \mathrm{M}$ Isoxaben treatment was assayed. As expected, Isoxaben induced ROP6 activity was greatly reduced in PMEI1OE line (Figure 5C). These results suggest that highly demethylesterified pectin is required for ROP6 signaling induced by mechanical stress. 


\section{A}

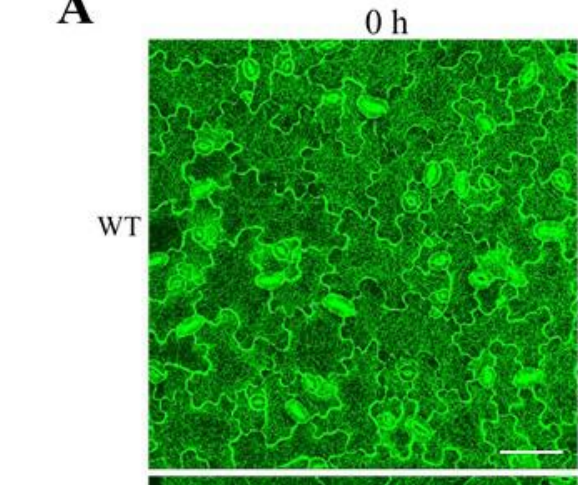

$0 \mathrm{~h}$

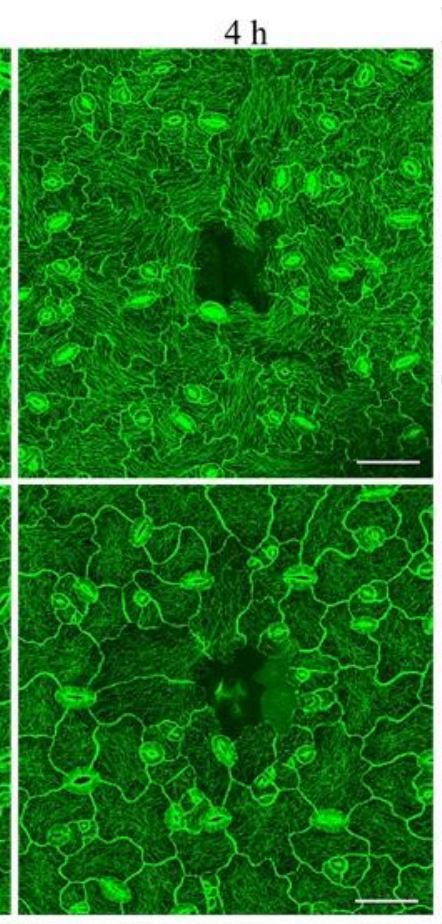

B

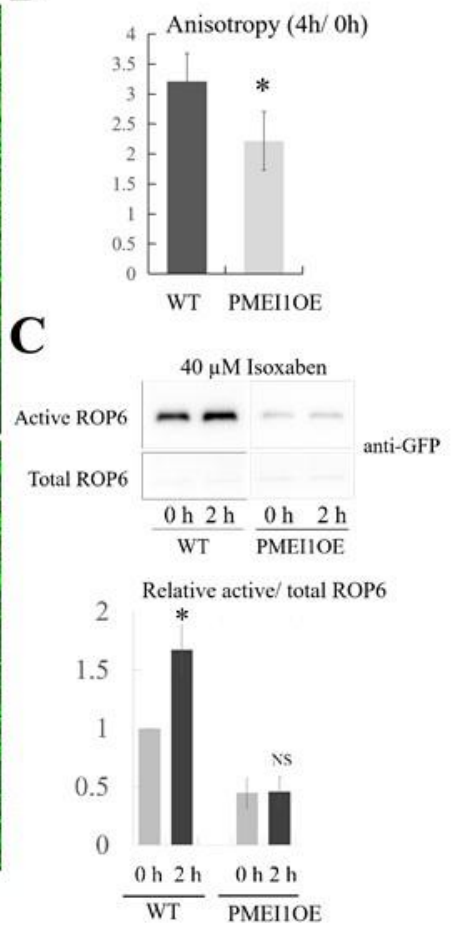

Figure 5. Demethylesterified pectin is important for the sensing of mechanical stress to induce MT rearrangement and ROP6 activation

(A) CMT rearrangement after single cell ablation on 3 DAG cotyledons from the GFPMAP4 and PMEIOE/GFP-MAP4 lines. Scale bar $=50 \mu \mathrm{m}$

(B) Quantification of MT Anisotropy in A. Two lays of PCs surrounding the ablated cell were chosen for quantification. Scale bar $=50 \mu \mathrm{m}$. Data are mean degrees from $>20$ independent cells \pm SE. Asterisks indicate the significant difference $\left({ }^{*} \mathrm{p}<0.05\right.$, Student's ttest) between the wild type and the mutants in above assays.

(C) ROP6 activity in 7 DAG seedlings of WT and PMEI1OE with and without $40 \mu \mathrm{M}$ Isoxaben treatment. Data are mean activity levels from three independent experiments \pm SD. *, $\mathrm{P}<0.05$; NS, no significance

\section{DISCUSSION}

Our current study together with the accompanying paper [46] provides strong support for the hypothesis that FER participates in sensing and/or transducing mechanical signal to directly regulate MT reorganization in the control of cell expansion and cell shape formation. First, we showed that both FER and its cell wall binding partner highly demethylesterified pectin are both required for MT re-orientation induced by mechanical stress. Second mechanical stress activates ROP6 signaling and this activation requires both FER and highly demethylesterified pectin. Third, the ROP6 signaling components, including ROP6 itself, its 
activator RopGEF14 that directly interacts with FER, and its effector RIC1 that directly regulate MT organization, are all important for the MT re-arrangement induced by mechanical stress (Figure 6) [46]. To our knowledge, the pectin/FER-ROP-MT pathway is the most complete mechano-transducing pathway connecting mechanical stress to the cellular responses described in plants to date. Nonetheless, the confirmation of FER's role as a direct mechanosensor requires single molecule mechanosensing assays in which mechanosensing activity can be measured when forces are applied to the single FER molecule.

Extracellular matrices (ECM) such as cell walls in plants and fungi have long been proposed to participate in mechanosensation, and this concept is well-established in animal systems [56, 57]. This concept involves the direct binding of an ECM component to the extracellular region of a transmembrane receptor, in such that the stiffness of ECM and changes in turgor pressure in the cell, could be mechanically monitored by the receptor via changes its conformation. However direct evidence for cell wall's role in the mechanotransduction and the identity of its transmembrane receptor partner remained elusive. As in animals, the cytoskeleton is also a central feature of the plant cell's response to mechanical stress [30], but how mechanical signals regulate the re-organization of the cytoskeleton is largely unknown. Therefore, our finding that the pectin-FER module is involved in mechanosensing and/or mechano-transduction and is directly linked to ROP6 signaling to the re-organization of cortical MTs is quite significant, as it provides important insights into the molecular mechanisms for mechanotransduction to regulate cell expansion and cell morphogenesis that relies on the mechanics of the cell wall in plants. Specifically, in PCs, the indenting regions are enriched in highly demethylesterified pectin making this region to experience mechanical stress differentially from lobing regions. Conceivably the pectin-FER senses the mechanical cue to promote cellulose microfibril deposition in the regions, re-enforcing them and enhancing the indentations (Figure 6). We propose that the pectin-FER system, by monitoring changes cell wall composition/modification and the resulting mechanical cues, feedback regulates the reconstruction of the cell wall, and consequently modulate cell shape formation, cell expansion, and cell wall integrity.

In addition to cell expansion and morphogenesis, FER has also been shown to maintain cell wall integrity under salt stress and in root hairs [45]. Several FER homologs in the CrRLK1L subfamily such as ANX1/2 and BUPS1/2 have been shown to maintain cell wall integrity in the rapidly growing cells such as pollen tubes. Interestingly BUPS1/CUP1 also binds pectin and participates in the sensing and/or signaling of mechanical stress to ROPs to control cell wall integrity of pollen tubes during their penetrative growth with the pistil [45, 58]. Hence the CrRLK1L subfamily of receptor kinases may have a widespread role in mechanosensation and/or mechano-transduction in plants. 


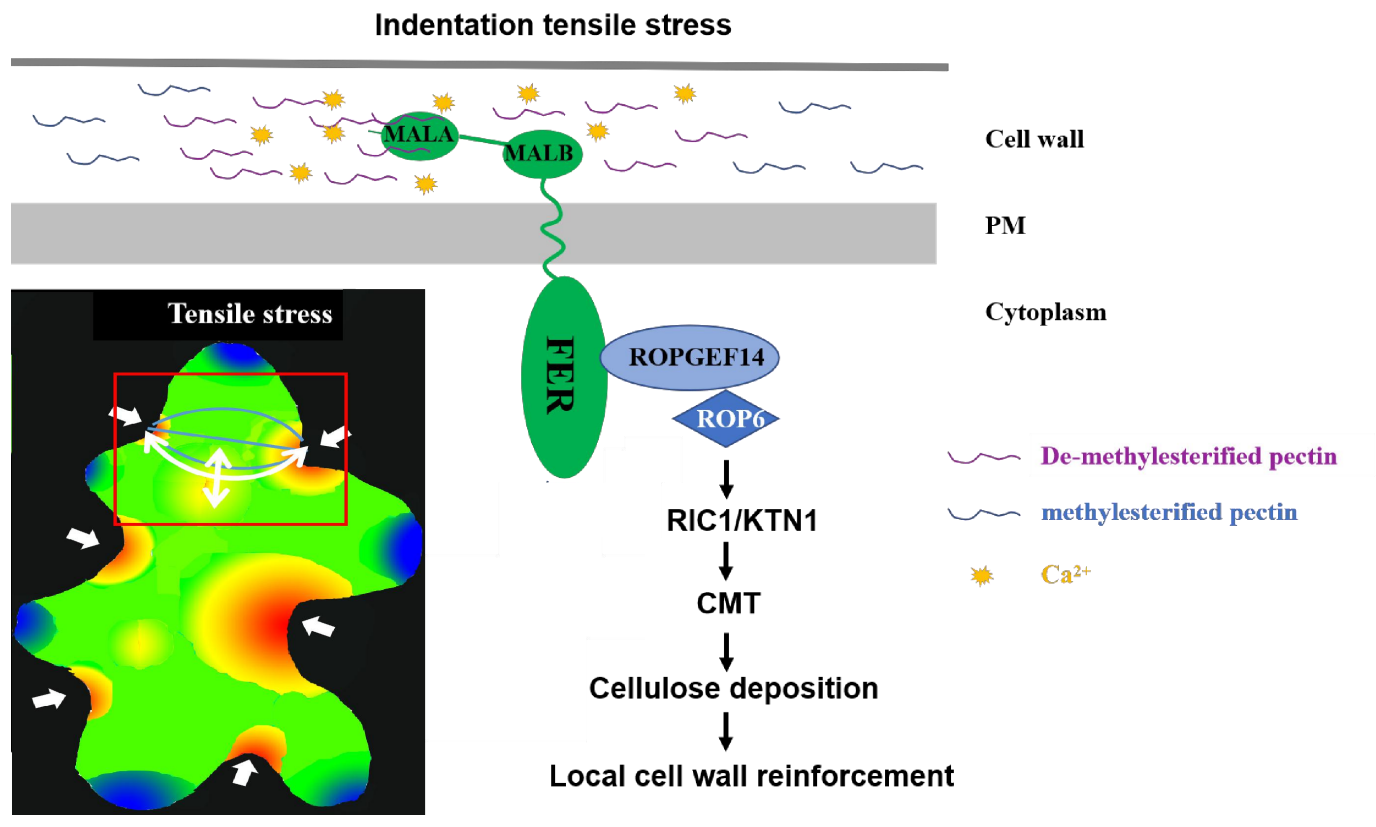

Figure 6. A working model for FER's function in sensing/signaling mechanical stress in PC indentation region to strengthen local cell wall by activating the ROP6 signaling pathway. Indentation regions of PC experience highest mechanical stress [33]. By FER binding to highly demethylesterified pectin, which preferentially accumulates in the indentation region, FER may sense this mechanical stress and activates the ROP6 signaling pathway by interacting with ROPGEF14. Active ROP6 then promotes the reorganization of cortical microtubule (CMT), which guilds cellulose deposition to strengthen local cell wall, leading to increased stiffness of the cell wall. Left bottom arrowheads indicate regions of highest stress magnitude in neck regions, blue lines indicate radial arrangement of CMT and white double arrow lines shows the tensile stress.

\section{METHODS}

\section{Plant Materials and Growth Conditions}

411 The fer-4 (GABI_GK106A06) and gef14-2 (Salk_064617C) were obtained from the Arabidopsis Biological Resource Center (ABRC) at Ohio State University, Columbus, OH. The PMEI1-OE were obtained from Vincenzo Lionetti (Sapienza University of Rome, Italy). GFP-TUBxrop6-1 and GFP-TUBxric1-1 were generated in our lab as described in [29]. The GFP-MAP4xfer-4, GFP-TUBxfer-4, GFP-ROP6xfer-4, GFP-ROP2xfer-4, GFPMAP4xPMEIIOE, GFP-ROP6xPMEIIOE, GFP-ROP6xgef14-2 and GFP-TUBxgef14-2 mutants were generated by genetic crosses and confirmed by genotyping or Western blotting. Arabidopsis plants were grown in soil (Sungro S16-281) in a growth room at $23^{\circ} \mathrm{C}, 40 \%$ 
seedlings, the seeds were surface sterilized with $50 \%$ (vol/vol) bleach for $10 \mathrm{~min}$, and then placed on the plates with $1 / 2$ MS medium containing $0.5 \%$ sucrose, and $1.0 \%$ agar at $\mathrm{pH} 5.7$.

\section{Mechanical perturbations}

Three types of mechanical perturbations (ablation, compression and Isoxaben treatment) were applied on pavement cells of Arabidopsis cotyledons. Single pavement cell from the same region of cotyledon was ablated by high-dosage UV light radiation (Confocal SP5) on the epidermis of cotyledons from seedlings. When the target cell was ablated, there would be a dark region with clear cell shape left. Ablation time depended on the stage of cotyledons. For the ablation of 1 DAG PCs, 10s were sufficient. For 3 DAG PCs, we applied 30-40s of UV irradiation. For 7 DAG PCs, the ablation time was increased to 60s. For compression, we first moved seedlings to a petri dish plate with absorbent paper (kept wet with liquid $1 / 2 \mathrm{MS}$ ) and placed a coverslip beside them. Then we applied compressive forces on the cotyledons by using a coverslip that was pressed on the surface of the cotyledons and kept in place using adhesive silicone applied on the margins. Then the samples were loaded for MT observation. To investigate the effects of compression on ROP activity, 3-weeks old leaves were collected and cultured in a plate with absorbent paper (kept wet with liquid $1 / 2$ MS). After $30 \mathrm{~min}$, four glass slides were placed on one half of a leaf as compression treatment, and the other half was free of external pressure as control. At various times, the glass slides were removed, and the leaves were dissected into two halves and immediately frozen in liquid nitrogen for ROP6 activity assay. For Isoxaben treatment on cotyledons for MT observation, seedlings were cultured in solid $1 / 2$ MS and then moved to a plate with absorbent paper (kept wet with liquid $1 / 2 \mathrm{MS}$ ) for $30 \mathrm{~min}$. The seedlings were then loaded for MT imaging on cotyledon PCs $(0 \mathrm{~h})$. After imaging these seedlings were transferred to a petri dish plate with absorbent paper, which was kept wet with liquid $1 / 2$ MS containing Isoxaben. After $4 \mathrm{~h}$ or $6 \mathrm{~h}$, the same cotyledons were loaded for MT imaging on the same regions imaged at $0 \mathrm{hr}$. For the analysis of Isoxaben treatment on ROP activities, seedlings were first cultured to $7 \mathrm{DAG}$ in half solid $1 / 2 \mathrm{MS}(0.4 \%$ agar $)$, and then transferred to regular liquid $1 / 2 \mathrm{MS}$. After $30 \mathrm{~min}$ incubation, the seedlings were transferred to liquid $1 / 2 \mathrm{MS}$ containing different concentrations of Isoxaben. Finally, the Isoxaben-treated seedlings were collected for ROP activity measurements.

\section{Quantitative analysis of cortical microtubule orientation.}

CMT of cotyledon PCs expressing MAP4-GFP were imaged using a Leica SP5 Laser Scanning Confocal. FibrilTool, an ImageJ plug-in to quantify fibrillar structures, was used to analyze the average anisotropy of MT [52]. The anisotropy score " 0 " indicates no order (isotropic arrays) and " 1 " indicates perfectly ordered (anisotropic arrays). For quantitative analysis of local cortical microtubule orientation in Figures $1 \mathrm{C}$ and 1D, only the indentation regions were selected with the Polygon tool for the analysis. Each data set was from the measurement of at least 20 cells collected from 3 different cotyledons from 3 individual seedlings. To examine the effect of mechanical stress on MT rearrangements, anisotropy of entire pavement cell before and after treatment was measured. For ablation treatment, two layers of PCs surrounding the ablated cell were used for anisotropy analysis. For 
compression and Isoxaben treatments, PCs at the same region of cotyledon (mid-region) were selected for anisotropy analysis.

\section{Measurement of ROP6 activity.}

Western blotting-based ROP activity measurements were performed as described in [24]. Total ROP6 and activated ROP6 proteins that were pulled down by MBP-RIC1 (GTP-bound ROP6) were detected by Western blotting analysis using anti-ROP6 or anti-GFP and horseradish peroxidase-conjugated rabbit antibodies. For ROP6 activity assays in 7 DAG seedlings, $0.2 \mathrm{~g}$ samples were used for each assay. For 3-week-old leaves, 0.4-0.5 g samples were collected for ROP activity measurements. Image $\mathrm{J}$ was used to quantify the mounts of ROPs.

\section{Acknowledgements}

We thank Olivier Hamant and members of the Yang laboratory for stimulating discussion and critical comments on this work. We thank Vincenzo Lionetti (Sapienza University of Rome, Italy) for providing PMEI1OE seeds. We appreciate microscopy assistance from the Institute of Integrative Genome Biology Microscopy Core Faculty (UC Riverside, US). This work is in part supported by a grant from National Natural Science Foundation of China to W.T. (No. 3150080116).

\section{Reference}

1. Chehab, E.W., Eich, E., and Braam, J. (2009). Thigmomorphogenesis: a complex plant response to mechano-stimulation. J Exp Bot 60, 43-56.

2. Mirabet, V., Das, P., Boudaoud, A., and Hamant, O. (2011). The role of mechanical forces in plant morphogenesis. Annu Rev Plant Biol 62, 365-385.

3. Monshausen, G.B., and Haswell, E.S. (2013). A force of nature: molecular mechanisms of mechanoperception in plants. J Exp Bot 64, 4663-4680.

4. Shih, H.W., Miller, N.D., Dai, C., Spalding, E.P., and Monshausen, G.B. (2014). The receptor-like kinase FERONIA is required for mechanical signal transduction in Arabidopsis seedlings. Curr Biol 24, 1887-1892.

5. Hamant, O., and Haswell, E.S. (2017). Life behind the wall: sensing mechanical cues in plants. BMC Biol 15, 59.

6. Voxeur, A., and Hofte, H. (2016). Cell wall integrity signaling in plants: "To grow or not to grow that's the question". Glycobiology 26, 950-960.

7. Veley, K.M., Marshburn, S., Clure, C.E., and Haswell, E.S. (2012). Mechanosensitive channels protect plastids from hypoosmotic stress during normal plant growth. Curr Biol 22, 408-413.

8. Hamilton, E.S., Schlegel, A.M., and Haswell, E.S. (2015). United in diversity: mechanosensitive ion channels in plants. Annu Rev Plant Biol 66, 113-137.

9. Monshausen, G.B., and Gilroy, S. (2009). Feeling green: mechanosensing in plants. Trends Cell Biol 19, 228-235.

10. Green, P.B. (1962). Mechanism for Plant Cellular Morphogenesis. Science 138, 1404-1405.

11. Ledbetter, M.C., and Porter, K.R. (1963). A "Microtubule" in Plant Cell Fine Structure. J Cell Biol 19, 239-250. 
12. Williamson, R.E. (1990). Alignment of cortical microtubules by anisotropic wall stresses. Functional Plant Biology 17, 601-613.

13. Schopfer, P. (2006). Biomechanics of plant growth. Am J Bot 93, 1415-1425.

14. Hamant, O., Heisler, M.G., Jonsson, H., Krupinski, P., Uyttewaal, M., Bokov, P., Corson, F., Sahlin, P., Boudaoud, A., Meyerowitz, E.M., et al. (2008). Developmental patterning by mechanical signals in Arabidopsis. Science 322, 1650-1655.

15. Hervieux, N., Dumond, M., Sapala, A., Routier-Kierzkowska, A.L., Kierzkowski, D., Roeder, A.H., Smith, R.S., Boudaoud, A., and Hamant, O. (2016). A Mechanical Feedback Restricts Sepal Growth and Shape in Arabidopsis. Curr Biol.

16. Hervieux, N., Tsugawa, S., Fruleux, A., Dumond, M., Routier-Kierzkowska, A.L., Komatsuzaki, T., Boudaoud, A., Larkin, J.C., Smith, R.S., Li, C.B., et al. (2017). Mechanical Shielding of Rapidly Growing Cells Buffers Growth Heterogeneity and Contributes to Organ Shape Reproducibility. Curr Biol 27, 3468-3479 e3464.

17. Verger, S., Long, Y., Boudaoud, A., and Hamant, O. (2018). A tension-adhesion feedback loop in plant epidermis. Elife 7.

18. Lin, W., and Yang, Z. (2020). Unlocking the mechanisms behind the formation of interlocking pavement cells. Current opinion in plant biology 57, 142-154.

19. Fu, Y., Li, H., and Yang, Z. (2002). The ROP2 GTPase controls the formation of cortical fine F-actin and the early phase of directional cell expansion during Arabidopsis organogenesis. Plant Cell 14, 777-794.

20. Fu, Y., Gu, Y., Zheng, Z., Wasteneys, G., and Yang, Z. (2005). Arabidopsis interdigitating cell growth requires two antagonistic pathways with opposing action on cell morphogenesis. Cell 120, 687-700.

21. Basu, D., Le, J., Zakharova, T., Mallery, E.L., and Szymanski, D.B. (2008). A SPIKE1 signaling complex controls actin-dependent cell morphogenesis through the heteromeric WAVE and ARP2/3 complexes. Proc Natl Acad Sci U S A 105, 4044-4049.

22. Fu, Y., Xu, T., Zhu, L., Wen, M., and Yang, Z. (2009). A ROP GTPase signaling pathway controls cortical microtubule ordering and cell expansion in Arabidopsis. Curr Biol 19, $1827-1832$.

23. Xu, T., Wen, M., Nagawa, S., Fu, Y., Chen, J.G., Wu, M.J., Perrot-Rechenmann, C., Friml, J., Jones, A.M., and Yang, Z. (2010). Cell surface- and rho GTPase-based auxin signaling controls cellular interdigitation in Arabidopsis. Cell 143, 99-110.

24. Xu, T., Dai, N., Chen, J., Nagawa, S., Cao, M., Li, H., Zhou, Z., Chen, X., De Rycke, R., Rakusova, H., et al. (2014). Cell surface ABP1-TMK auxin-sensing complex activates ROP GTPase signaling. Science 343, 1025-1028.

25. Kropf, D.L., Bisgrove, S.R., and Hable, W.E. (1998). Cytoskeletal control of polar growth in plant cells. Curr Opin Cell Biol 10, 117-122.

26. Wasteneys, G.O. (2000). The cytoskeleton and growth polarity. Curr Opin Plant Biol 3, 503511.

27. Martin, C., Bhatt, K., and Baumann, K. (2001). Shaping in plant cells. Curr Opin Plant Biol 4, 540-549.

28. Yang, Z. (2008). Cell polarity signaling in Arabidopsis. Annu Rev Cell Dev Biol 24, 551575.

29. Lin, D., Cao, L., Zhou, Z., Zhu, L., Ehrhardt, D., Yang, Z., and Fu, Y. (2013). Rho GTPase signaling activates microtubule severing to promote microtubule ordering in Arabidopsis. Curr Biol 23, 290-297.

30. Sampathkumar, A., Krupinski, P., Wightman, R., Milani, P., Berquand, A., Boudaoud, A., Hamant, O., Jonsson, H., and Meyerowitz, E.M. (2014). Subcellular and supracellular mechanical stress prescribes cytoskeleton behavior in Arabidopsis cotyledon pavement cells. Elife 3, e01967. 
31. Majda, M., Grones, P., Sintorn, I.M., Vain, T., Milani, P., Krupinski, P., Zagorska-Marek, B., Viotti, C., Jonsson, H., Mellerowicz, E.J., et al. (2017). Mechanochemical Polarization of Contiguous Cell Walls Shapes Plant Pavement Cells. Dev Cell 43, 290-304 e294.

32. Jacques, E., Verbelen, J.P., and Vissenberg, K. (2013). Mechanical stress in Arabidopsis leaves orients microtubules in a 'continuous' supracellular pattern. BMC Plant Biol 13, 163.

33. Bidhendi, A.J., and Geitmann, A. (2019). Geometrical Details Matter for Mechanical Modeling of Cell Morphogenesis. Dev Cell 50, 117-125 e112.

34. Sapala, A., Runions, A., Routier-Kierzkowska, A.L., Das Gupta, M., Hong, L., Hofhuis, H., Verger, S., Mosca, G., Li, C.B., Hay, A., et al. (2018). Why plants make puzzle cells, and how their shape emerges. Elife 7.

35. Escobar-Restrepo, J.M., Huck, N., Kessler, S., Gagliardini, V., Gheyselinck, J., Yang, W.C., and Grossniklaus, U. (2007). The FERONIA receptor-like kinase mediates male-female interactions during pollen tube reception. Science 317, 656-660.

36. Keinath, N.F., Kierszniowska, S., Lorek, J., Bourdais, G., Kessler, S.A., Shimosato-Asano, H., Grossniklaus, U., Schulze, W.X., Robatzek, S., and Panstruga, R. (2010). PAMP (pathogen-associated molecular pattern)-induced changes in plasma membrane compartmentalization reveal novel components of plant immunity. J Biol Chem 285, 3914039149.

37. Kessler, S.A., Shimosato-Asano, H., Keinath, N.F., Wuest, S.E., Ingram, G., Panstruga, R., and Grossniklaus, U. (2010). Conserved molecular components for pollen tube reception and fungal invasion. Science 330, 968-971.

38. Guo, H., Nolan, T.M., Song, G., Liu, S., Xie, Z., Chen, J., Schnable, P.S., Walley, J.W., and Yin, Y. (2018). FERONIA Receptor Kinase Contributes to Plant Immunity by Suppressing Jasmonic Acid Signaling in Arabidopsis thaliana. Curr Biol 28, 3316-3324 e3316.

39. Guo, H., Li, L., Ye, H., Yu, X., Algreen, A., and Yin, Y. (2009). Three related receptor-like kinases are required for optimal cell elongation in Arabidopsis thaliana. Proc Natl Acad Sci U S A 106, 7648-7653.

40. Deslauriers, S.D., and Larsen, P.B. (2010). FERONIA is a key modulator of brassinosteroid and ethylene responsiveness in Arabidopsis hypocotyls. Mol Plant 3, 626-640.

41. Duan, Q., Kita, D., Li, C., Cheung, A.Y., and Wu, H.M. (2010). FERONIA receptor-like kinase regulates RHO GTPase signaling of root hair development. Proc Natl Acad Sci U S A $107,17821-17826$.

42. Huang, G.Q., Li, E., Ge, F.R., Li, S., Wang, Q., Zhang, C.Q., and Zhang, Y. (2013). Arabidopsis RopGEF4 and RopGEF10 are important for FERONIA-mediated developmental but not environmental regulation of root hair growth. New Phytol 200, 10891101.

43. Li, C., Yeh, F.L., Cheung, A.Y., Duan, Q., Kita, D., Liu, M.C., Maman, J., Luu, E.J., Wu, B.W., Gates, L., et al. (2015). Glycosylphosphatidylinositol-anchored proteins as chaperones and co-receptors for FERONIA receptor kinase signaling in Arabidopsis. Elife 4.

44. Yu, F., Li, J., Huang, Y., Liu, L., Li, D., Chen, L., and Luan, S. (2014). FERONIA receptor kinase controls seed size in Arabidopsis thaliana. Mol Plant 7, 920-922.

45. Feng, W., Kita, D., Peaucelle, A., Cartwright, H.N., Doan, V., Duan, Q., Liu, M.C., Maman, J., Steinhorst, L., Schmitz-Thom, I., et al. (2018). The FERONIA Receptor Kinase Maintains Cell-Wall Integrity during Salt Stress through Ca(2+) Signaling. Curr Biol 28, 666-675 e665.

46. Lin, W., Tang, W., Anderson, C.T., Yang, Z. (2018). FERONIA's sensing of cell wall pectin activates ROP GTPase signaling in Arabidopsis.

47. Mammoto, T., Mammoto, A., and Ingber, D.E. (2013). Mechanobiology and developmental control. Annu Rev Cell Dev Biol 29, 27-61.

48. Verna, J., Lodder, A., Lee, K., Vagts, A., and Ballester, R. (1997). A family of genes required for maintenance of cell wall integrity and for the stress response in Saccharomyces cerevisiae. Proc Natl Acad Sci U S A 94, 13804-13809. 
604

605

606

607

608

609

610

611

612

613

614

615

616

617

618

619

620

621

622

623

624

625

626

627

628

629

630

631

632

633

634

635

636

637

638

639

640

641

642

643

644
49. Philip, B., and Levin, D.E. (2001). Wsc1 and Mid2 are cell surface sensors for cell wall integrity signaling that act through Rom2, a guanine nucleotide exchange factor for Rho1. Mol Cell Biol 21, 271-280.

50. Straede, A., and Heinisch, J.J. (2007). Functional analyses of the extra- and intracellular domains of the yeast cell wall integrity sensors Mid2 and Wsc1. FEBS Lett 581, 4495-4500.

51. Dupres, V., Alsteens, D., Wilk, S., Hansen, B., Heinisch, J.J., and Dufrene, Y.F. (2009). The yeast Wsc1 cell surface sensor behaves like a nanospring in vivo. Nat Chem Biol 5, 857-862.

52. Boudaoud, A., Burian, A., Borowska-Wykret, D., Uyttewaal, M., Wrzalik, R., Kwiatkowska, D., and Hamant, O. (2014). FibrilTool, an ImageJ plug-in to quantify fibrillar structures in raw microscopy images. Nat Protoc 9, 457-463.

53. Heisler, M.G., Hamant, O., Krupinski, P., Uyttewaal, M., Ohno, C., Jonsson, H., Traas, J., and Meyerowitz, E.M. (2010). Alignment between PIN1 polarity and microtubule orientation in the shoot apical meristem reveals a tight coupling between morphogenesis and auxin transport. PLoS Biol 8, e1000516.

54. Uyttewaal, M., Burian, A., Alim, K., Landrein, B., Borowska-Wykret, D., Dedieu, A., Peaucelle, A., Ludynia, M., Traas, J., Boudaoud, A., et al. (2012). Mechanical stress acts via katanin to amplify differences in growth rate between adjacent cells in Arabidopsis. Cell 149, 439-451.

55. Landrein, B., and Hamant, O. (2013). How mechanical stress controls microtubule behavior and morphogenesis in plants: history, experiments and revisited theories. Plant J 75, 324-338.

56. Broders-Bondon, F., Nguyen Ho-Bouldoires, T.H., Fernandez-Sanchez, M.E., and Farge, E. (2018). Mechanotransduction in tumor progression: The dark side of the force. J Cell Biol 217, 1571-1587.

57. Fattet, L., Jung, H.Y., Matsumoto, M.W., Aubol, B.E., Kumar, A., Adams, J.A., Chen, A.C., Sah, R.L., Engler, A.J., Pasquale, E.B., et al. (2020). Matrix Rigidity Controls EpithelialMesenchymal Plasticity and Tumor Metastasis via a Mechanoresponsive EPHA2/LYN Complex. Dev Cell 54, 302-316 e307.

58. Zhou, X., Lu, J., Zhang, Y., Guo, J. Lin, W., Van Norman, J., Qin, Y., Zhu, X., Yang, Z. (2020). A membrane receptor-mediated mechanosensing system maintains cell integrity during invasive growth. 

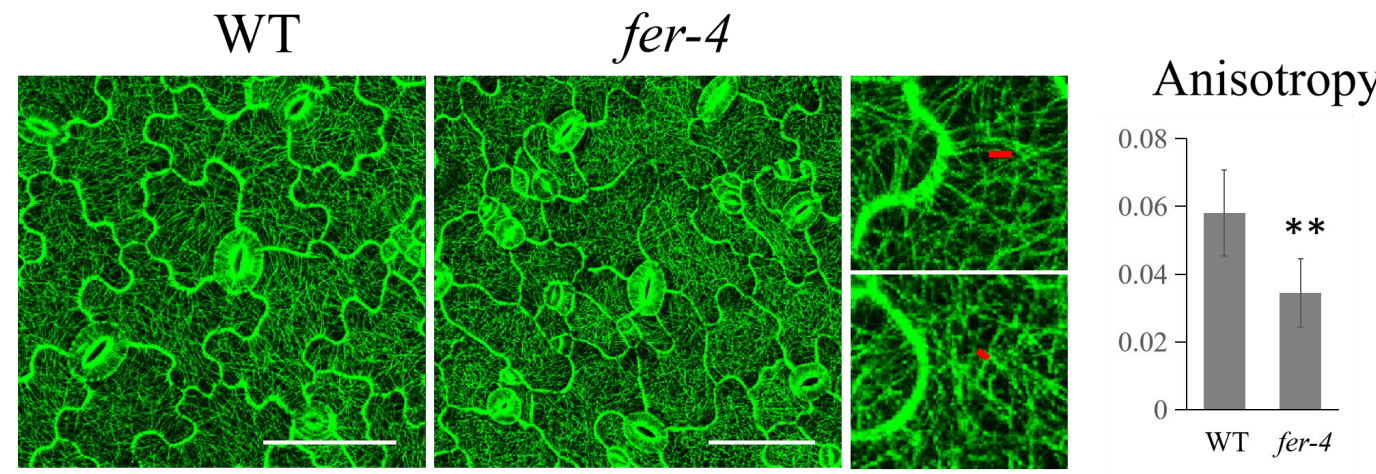

Fig. S1 The radial distribution of cortical MT (microtubules) was greatly reduced in indentations of fer-4 PCs (2 DAG). Right showed PC cortical MT of wild-type (GFP-MAP4) and the fer-4 mutant (fer-4xGFP-MAP4) grown in $1 / 2 \mathrm{MS}$ with $1.0 \%$ agar. Bar, $50 \mu \mathrm{m}$. Left was the magnified PC indentation regions. The degree of cortical MT anisotropy was quantified. Red lines

were generated by FibrilTool, an Image J plug-in to quantify fibrillar structures in raw microscopy images. the angle of red line represents the average orientation of the array and the length of red line is proportional to the array anisotropy. Data are mean degrees from $>20$ independent cells \pm SE. ${ }^{* *} p<0.01$

A

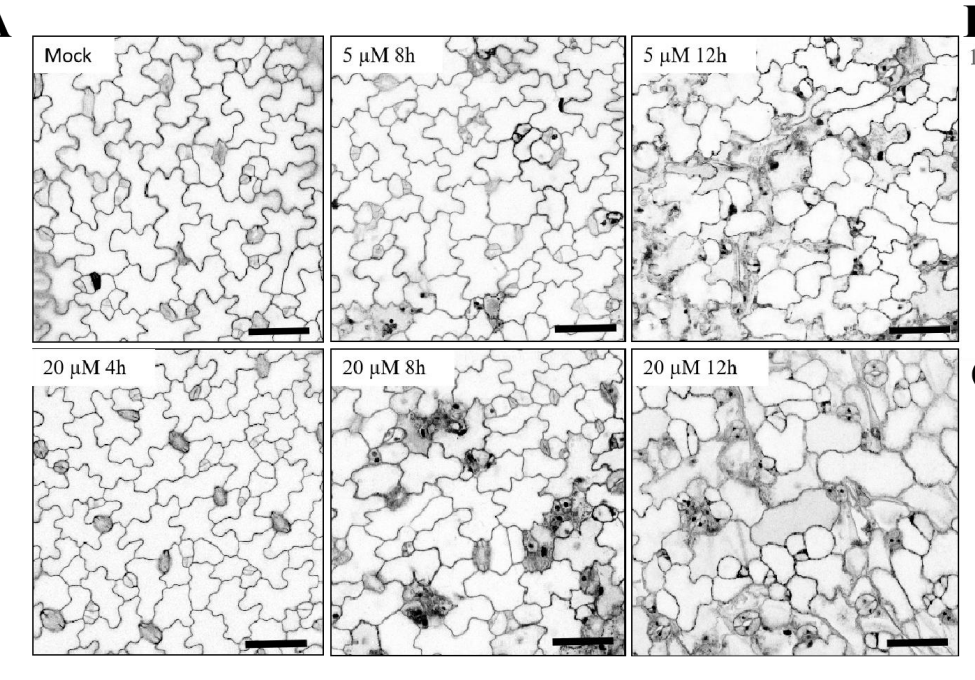

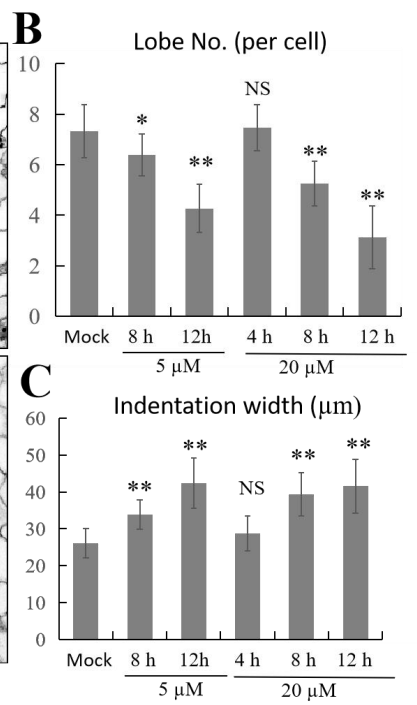

656

657

658

659

660

661

662

Fig. S2 Isoxaben Treatment induces pavement cell shape change.

(A) PC phenotypes of wild-type (Col-0) treated with different concentrations (mock, $5 \mu \mathrm{M}, 20 \mu \mathrm{M}$ of Isoxaben in different times $(0 \mathrm{~h}, 4 \mathrm{~h}, 8 \mathrm{~h}, 12 \mathrm{~h})$. Scale bar, $50 \mu \mathrm{m}$.

(B) PC lobe number quantification result showed $>5 \mu \mathrm{M}$ Isoxaben significantly inhibit lobe formation. Data are represented as mean $\pm \mathrm{SE} .{ }^{*} p<0.05 ;{ }^{* *} p<0.01$; Student's $t$-test

(C) PC indentation width quantification result showed $>5 \mu \mathrm{M}$ Isoxaben significantly inhibit neck formation. Data are represented as mean $\pm \mathrm{SE} . \quad{ }^{* *} p<0.01$; Student's $t$-test 
A

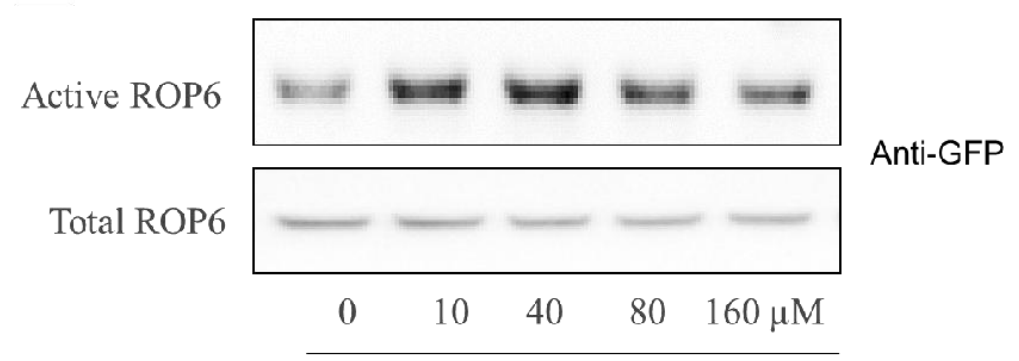

Isoxaben $2 \mathrm{~h}$

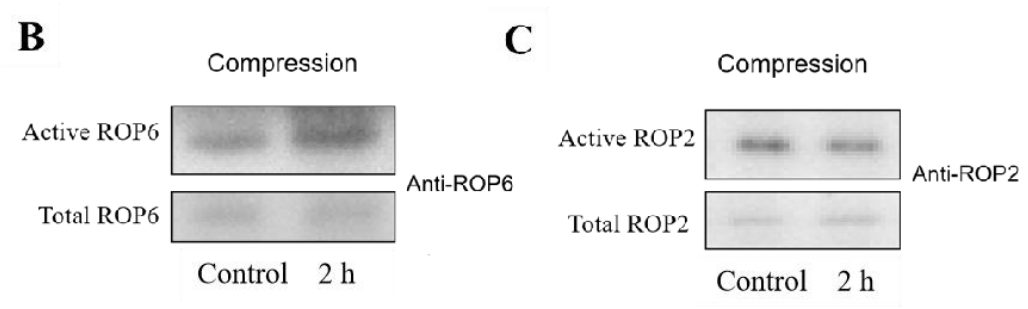

664 Fig. S3 ROP6 can be activated by different concentrations of Isoxaben and compression.

665 (A) ROP6 activity in 7 DAG seedlings of GFP-ROP6 with different concentrations of isoxaben.

666 Seedlings of GFP-ROP6 were incubated in 1/2MS with 10, $20,40,80,160 \mu \mathrm{M}$ Isoxaben. The amount of

667 active ROP6 increased most in 10, $40 \mu \mathrm{M}$ Isoxaben treated seedlings comparing to 80 and $160 \mu \mathrm{M}$

668 Isoxaben treatment

669 (B) ROP6 is activated by compression. 3 weeks leaves of Col-0 were used for 2 hours compression treatment. Active and total ROP6 were detected by western blots using Anti-ROP6.

671 (C) 2 hours compression have no effects on ROP2 activation. 3 weeks leaves of Col-0 were used for 2 hours compression treatment. Active and total ROP 2 were detected by western blots using Anti-ROP2.
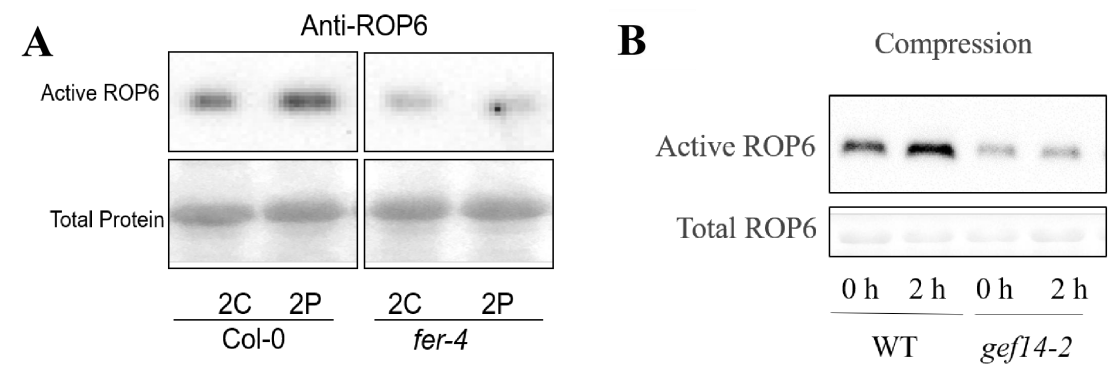

674 Fig S4 ROP6 can be obviously activated by compression in Col-0 but not fer-4 and gef14-2.

675 (A) Compression induced ROP6 activation is lost in fer-4. C, control; P, pressure

676 (B) ROPGEF14 is required for compression induced ROP6 activation. 
A
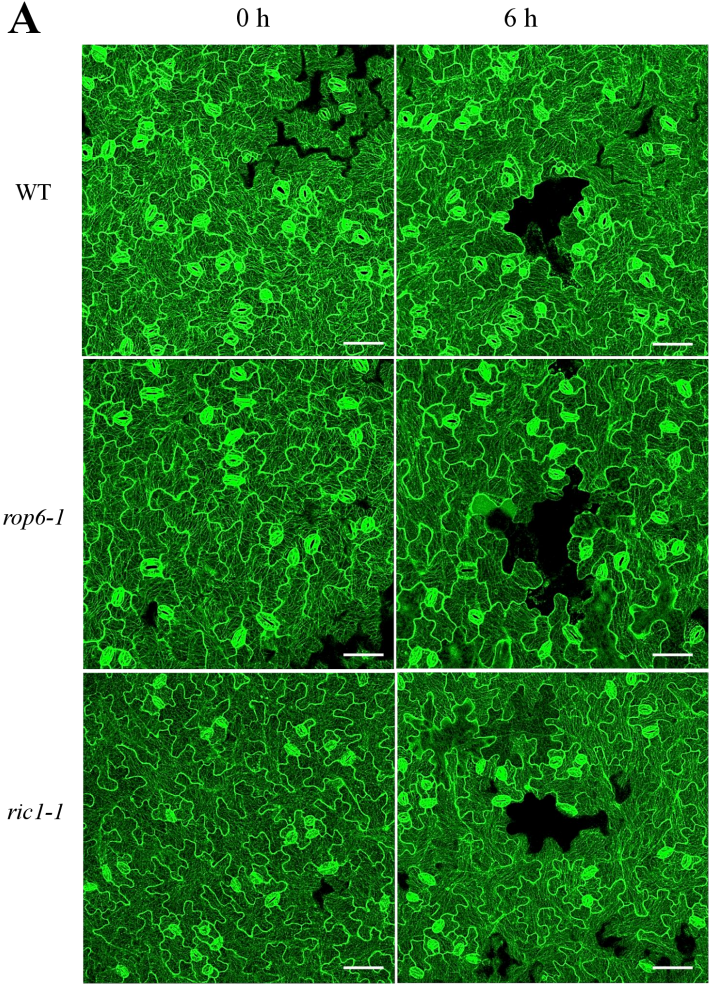

B
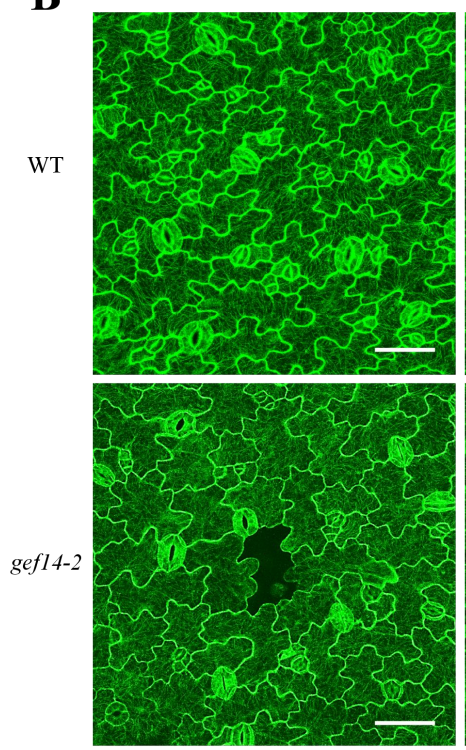

C

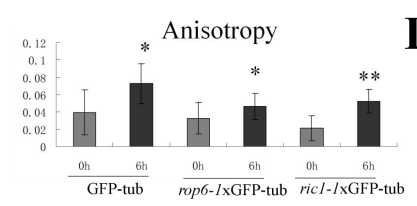

$4 \mathrm{~h}$
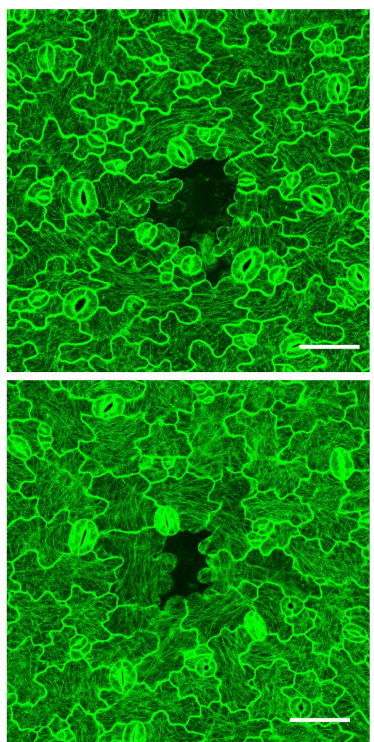

D

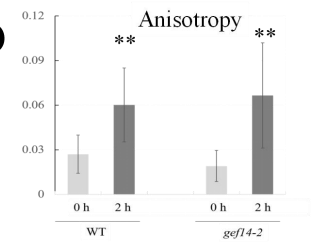

Fig. S5 Early stage of PCs (3 DAG) of rop6-1, ric1-1 and gef14-2 showed normal sensitivity to ablation in terms of $M T$ rearrangement.

680

(A) MT rearrangements after single cell ablation in WT, rop6-1 and ric1-1 PCs. 7 DAG cotyledon grown in $1 / 2 \mathrm{MS}$ with normal concentration of agar $(1.0 \%)$ were observed in Confocal SP5. Red line represents the anisotropy of MT. Two layers of PCs around ablation point were quantified. Bar, $50 \mu \mathrm{m}$. (B) MT rearrangements after single cell ablation in WT and gef14-2 PCs. 3 DAG cotyledon grown in $1 / 2$ MS with normal concentration of agar $(1.0 \%)$ were observed in Confocal SP5. Red line represents the anisotropy of MT. Two layers of PCs around ablation point were quantified. Bar, $50 \mu \mathrm{m}$. rearrangement in WT PC after ablation. In normal concentration of agar, both rop6-1 and ricl-1 showed no significant differences comparing to WT in terms of anisotropy change. Data are mean degrees from $>40$ independent cells \pm SE. ${ }^{*}, \mathrm{p}<0.05 ;^{* *}, \mathrm{p}<0.01$; NS, no significance

690 